\title{
Association between racial discrimination and health-related quality of life and the impact of social relationships
}

\author{
Genevieve Bergeron $^{1,2}$ (D) - Nneka Lundy De La Cruz ${ }^{1} \cdot$ L. Hannah Gould ${ }^{1} \cdot$ Sze Yan Liu ${ }^{1} \cdot$ Amber Levanon Seligson $^{1}$
}

Accepted: 7 May 2020 / Published online: 22 May 2020

(c) Springer Nature Switzerland AG 2020

\begin{abstract}
Purpose Interpersonal racial discrimination is associated with poor health. Social relationships may moderate the impact of discrimination and represent modifiable behaviors that can be targeted by public health interventions. We described citywide associations between self-reported racial discrimination and health-related quality of life among the overall New York City (NYC) adult residential population and by four main race/ethnicity groups and explored whether social relationships moderated health effects of discrimination.

Methods We analyzed cross-sectional survey data from 2335 adults weighted to be representative of the NYC population. We measured exposures to lifetime interpersonal racial discrimination in nine domains using a modified version of the Experiences of Discrimination scale. We performed unadjusted and adjusted regression analyses on four self-rated healthrelated quality of life outcomes including general health, physical health, mental health, and limitations from physical or mental health.

Results Overall, 47\% [95\% CI 44.5, 50.3] of respondents reported having experienced racial discrimination in at least one domain. In the overall population, significant associations with racial discrimination were noted in adjusted models for poor physical health, poor mental health, and limitations by poor physical and mental health. Among those exposed to racial discrimination, the risk of experiencing poor mental health was lower among those who had contact with family or friends outside their household at least once a week, compared with those who had less frequent social contact.

Conclusion This study provides evidence that social relationships may moderate the impact of racial discrimination on mental health and should be integrated into health promotion efforts.
\end{abstract}

Keywords Health-related quality of life $\cdot$ Racial discrimination $\cdot$ Social relationships $\cdot$ Race/ethnicity $\cdot$ Racism $\cdot$ Social determinants of health

\section{Introduction}

Experiencing interpersonal racial discrimination is associated with poor health for an array of health outcomes including hypertension [1], obesity [2], breast cancer [3], asthma [4], mental health [5], and mortality [6]. The association persists even when adjusting for socioeconomic status [7].

Genevieve Bergeron

gbergeron@cdc.gov

1 New York City Department of Health and Mental Hygiene, New York, New York, United States

2 Division of Scientific Education and Professional Development, Epidemic Intelligence Service, Centers for Disease Control and Prevention, Atlanta, Georgia, United States
The underlying physiologic mechanisms have not been fully explained. However, research indicates metabolic pathways such as increased inflammation [8,9], increased stress on biological systems [10-12], oxidative stress [13], systemic aging [14], poor sleep [15, 16], and engagement in unhealthy behaviors might all contribute [17, 18].

Social relationships are an important social determinant of health. People who are more socially isolated and have fewer social interactions with others are more likely to die prematurely and have worse mental health and physical health [19-21]. Although precise mechanisms are not clear, scientists postulate that social relationships might have a direct impact on health or buffer the impact of stressors by providing emotional or informational resources [22, 23].

Although a substantial body of work indicates that racial discriminiation is adversely associated with poor health 
outcomes, limited research has examined potential effect measure modifiers. Lewis and colleagues in a review of self-reported discrimination and health suggested social and emotional support as an emerging factor that could act as a "buffer" from the effects of discrimination on health [24]. Prior research explored different concepts related to social relationships when examining the impact of racial discrimination on health such as seeking support when faced with discrimination, but findings have been mixed, as outlined by Brondolo and colleagues [25]. An important challenge to studying the impact of social relationships is the heterogeneity of concepts and measurements, which range from qualitative assessment of emotional support to quantitative description of social networks [26]. In a study from Finch and colleagues among Latinos in California, discrimination was associated with poor self-rated physical health only among those lacking social support [27]. A study of young African American adults in the rural southern United States indicated that emotional support might reduce the impact of racial discrimination on biological stress-regulation systems [11].

We sought to describe the associations between selfreported racial discrimination and health-related quality of life among a large, diverse urban population overall and by four main race/ethnicity groups, and to explore whether social relationships moderate negative health effects of racial discrimination.

\section{Methods}

We analyzed data from the 2017 NYC Department of Health and Mental Hygiene (DOHMH) Social Determinants of Health (SDH) survey, a cross-sectional survey. Participants were non-institutionalized adults living in NYC. Race/ethnicity was categorized as white, black, Latino of any race, Asian/Pacific Islander (PI) and other or multiple races.

The survey combined two sampling methods that included random digit dialing of landline and cell telephone numbers and address-based sampling. Both methods were used in order to investigate survey methodological questions regarding response rates and response bias.

In total, 47,625 randomly selected working landline numbers were dialed. Randomly selected NYC cellular numbers were pre-screened to remove inactive numbers and supplemented with a sample of non-NYC cellular numbers with NYC ZIP code billing addresses for a total of 40,575 cellular telephone numbers. Telephone respondents could be interviewed in English, Spanish, Russian, Mandarin, or Cantonese using a computer-assisted technology interviewing system. Data collected from telephone interviews were adjusted for the initial probability of selection, dual cell phone and landline use, and non-response. Data collected from landline interviews were additionally adjusted for the probability of respondent selection in a household with multiple adults and for respondents in a household with two or more landlines. In total, 1433 responses were collected from random digit dialing.

Address-based sampling was done by randomly selecting NYC postal addresses from the U.S. Postal Service Computerized Delivery Sequence File. Address-based interviews were adjusted for the probability of unit selection, probability of selection in a household with multiple adults, and non-response. The survey was available only in English using pencil-and-paper and with an option to complete on the Internet. The sample included 6152 units and 902 responses were completed.

Although 47,625 landline and 40,575 cell phone numbers were dialed, many of those phone numbers were determined to be ineligible or were of unknown eligibility. For each sample, the eligibility of sampled records where eligibility status was able to be determined as either eligible or ineligible was used to estimate the number of eligible records within all records of unknown eligibility. This was then used in the denominator for calculating response rates. The combined response rate for both telephone and mail-based survey was $11.6 \%$ and the cooperation rate was $80.4 \%$ based on response rate \#3 in a modified version of the American Association for Public Opinion Research's (AAPOR) Standard Definitions and Response Rate Calculator V4.0 for dual frame random digit dialing and for mailing to unnamed persons [28].

The total of 2335 interviews were weighted to be representative of the NYC population of residential adults according to the 2015 U.S. Census Bureau American Community Survey estimate (6,585,635 adults). NYC's DOHMH Institutional Review Board approved this survey. This analysis was reviewed by CDC for human subject protection and was determined to be non-research.

The exposure variable measured self-reported lifetime interpersonal discrimination based on race, ethnicity, or color in nine domains among all race/ethnicity groups. Whites-a group typically considered the majority in the USA-was assessed for racial discrimination as part of a complete analysis of all race/ethnicity groups as was performed in other similar studies $[6,9,17,18,29-32]$. We used a modified version of the 9-item survey instrument Experiences of Discrimination developed by Dr. Nancy Krieger and validated in diverse populations [33]. The questions asked were: "Have you ever experienced discrimination, been prevented from doing something or been hassled or made to feel inferior in any of the following situations because of your race, ethnicity, or color... at school?, getting a job?, at work?, getting housing?, getting healthcare?, getting service in a store or restaurant?, getting credit, bank loans, or a mortgage?, on the street or in a public setting?, 
from the police or in the courts?" The original survey instrument had frequency-graded answers, including never, once, two to three times, four or more times. NYC's 2017 SDH survey Experiences of Discrimination scale used a dichotomous answer option (yes or no) for each of the nine domains.

Experiences with racial discrimination in different domains varied among race/ethnicity groups (data not shown). In order to determine whether certain race/ethnicity groups were more likely to experience discrimination than others, we conducted $t$-tests in SUDAAN and generated $p$ values to assess whether differences were statistically significant. Blacks and Latinos reported experiencing racial discrimination more frequently than whites on all domains. Asian/PIs were more likely than whites to report experiencing racial discrimination at school, while getting a job, at work, while getting housing, while getting services in a store or restaurant, and in a public setting. Blacks were more likely than Latinos to report experiencing discrimination at school, while getting a job, while getting housing, while getting service in a store or restaurant, and from police or in the courts. Blacks were more likely than Asian/PIs to report experiencing discrimination while getting a job, while getting housing, while getting service in a store or restaurant, while getting credit, bank loans or a mortgage, and from police or in the courts. All domains of racial discrimination were weighted equally and participants were given one point per domain experienced in their lifetime (range 0-9). Categories for exposure to racial discrimination were generated using three categories including having experienced zero domains, having experienced one to two domains, or having experienced three or more domains. We excluded four participants because respondents answered "don't know" or did not answer all nine domains of the question.

The outcome variables were the 4-item health-related quality of life (HrQOL-4) questions from the healthy days core module [34].

HrQOL \#1: "Would you say that in general your health is excellent, very good, good, fair or poor?"

HrQOL \#2: "Now thinking about your physical health, which includes physical illness and injury, how many days during the past 30 days was your physical health not good?"

HrQOL \#3: "Now thinking about your mental health, which includes stress, depression, and problems with emotions, how many days during the past 30 days was your mental health not good?"

HrQOL \#4: "During the past 30 days, for about how many days did poor physical or mental health keep you from doing your usual activities, such as self-care, work, or recreation?"

The HrQOL-4 questions are widely used in different population health surveys including the U.S. Behavioral Risk Factor Surveillance System and have been validated in various adult residential populations [35]. HrQOL \#1 was dichotomized into two categories for the analysis, as previously done in other studies, because of the low number of responses at the extreme ends of the spectrum within race/ethnicity groups $[34,36]$. It included excellent, very good, or good versus poor or fair. HrQOL \#2-4 measured unhealthy days during the past 30 days and were used without categorization.

Using the framework proposed by Valtorta et al. [26], we used a question that assessed a structural aspect of social relationships without requiring the respondent to assess the availability or adequacy of the relationship: "In the past 30 days, how often have you gotten together with at least one friend or family member, aside from those you live with?" Possible answers were almost every day, about once a week, a few times a month or not in the past 30 days, and were dichotomized between low-frequency social contact (i.e., a few times a month or less) and high-frequency social contact (i.e., about once a week or more).

We created an aggregate measure of material hardship to assess economic status because $11.5 \%$ reported not knowing or declined to answer information needed to calculate the household poverty level. In addition, income is often insufficient to assess poverty levels in NYC, because of the high cost of housing but also the availability of different benefit programs [37]. Material hardship was a dichotomous variable with an answer of yes being defined as meeting any of the following characteristics: difficulty affording basic items; difficulty affording food [38], difficulty affording rent [39], using a food assistance program, or not having enough money to make ends meet [40].

Statistical analyses were performed in SAS® 9.4 and SAS-callable SUDAAN® version 11.0.1 (SAS Institute, Inc., Cary, North Carolina). We described the percentage of participants who experienced racial discrimination by age group, race/ethnicity, sex, marital status, nativity, education, employment, material hardship, health insurance status, and frequency of social contact. We performed a $t$-test in SUDAAN for each socio-demographic variable to assess whether the percentage who had experienced racial discrimination was statistically different within each variable. We also described health outcomes by exposure categories. We conducted bivariate regression analyses of the racial discrimination exposure on the four HrQOL outcomes. The category of having experienced zero domains of racial discrimination was the reference group. Multivariable regressions generated model-adjusted relative risks (RR). Approximately $4 \%$ of respondents were excluded from the multivariable models because of a missing value in at least one variable. For HrQOL \#1, we used logistic regression because of the dichotomous self-rated health question. For HrQOL \#2-4, we used Poisson regression due to the outcomes that were counts of days. All associations are reported in RRs with lower and upper 95\% CIs. We analyzed participants overall and within race/ethnicity strata. 
Sex, age, and nativity were included in the adjusted model based on a priori assumptions. We also explored which socioeconomic factors, if any, to include in the adjusted model. Socioeconomic status (SES) covariates are possible intermediate variables in the causal pathway between experiencing racial discrimination and poor health. However, SES is also a strong determinant of poor health independently of experiencing racial discrimination. As previously described by Williams and colleagues [41], we sought to facilitate understanding of the association between experiencing racial discrimination and health by adjusting for socioeconomic covariates even if those covariates did not technically meet the definition of a confounder. We performed sensitivity analyses for education, employment, material hardship, and health insurance and included in the adjusted model any covariate with a greater than $10 \%$ impact on the estimate [42]. Only material hardship met this criterion. Additionally, adjusting for race in the overall population did not have a significant impact on the estimates (data not shown) and therefore all models were adjusted for the same covariates of sex, age, nativity, and material hardship.

We tested interaction between social relationships and having experienced racial discrimination by adding to the adjusted models an interaction term for those two variables. For models where the interaction term was significant $(p<0.05)$, we stratified the models by the social relationship variable to confirm direction of the association.

\section{Results}

Forty-seven percent [95\% CI 44.5, 50.3] of respondents reported experiences of racial discrimination in at least one domain. Reports of racial discrimination varied most widely by age, race/ethnicity, and material hardship status. Blacks (65.2\% [95\% CI 59.4, 70.6]), Asian/PIs (52.7\% [95\% CI 44.4, 60.8]), and Latinos (52.1\% [95\% CI 46.4, 57.7]) more frequently reported experiences of racial discrimination compared with whites (29.2\% [95\% CI 24.9, 33.8]). Similarly, non-whites more frequently reported experiencing three or more domains of racial discrimination than whites. Among respondents aged $\geq 65$ years, $29.3 \%$ [95\% CI 24.4, 34.8] reported experiencing racial discrimination, compared with $48.0 \%$ [95\% CI 28.7, 57.4] of people aged 18-24 years old. Among those who reported experiences of material hardship, 60.6\% [95\% CI 55.9, 65.0] reported experiences of racial discrimination, compared with $37.9 \%$ [95\% CI 34.5 , 41.4] among those who did not report experiences of material hardship (Table 1).

In the adjusted models for the overall population, there were no significant associations between exposure to racial discrimination and self-rated general health (Table 2) but significant associations were noted for self-rated poor physical health (Table 3), poor mental health (Table 4), and limitations by poor physical and mental health (Table 5). Compared with those who did not experience any racial discrimination, those who experienced three or more domains of racial discrimination had, out of the last 30 days, 1.40 [95\% CI 1.08-1.80] more days when their physical health was not good, 1.63 [95\% CI 1.26-2.12] more days when their mental health was not good and 1.74 [95\% CI 1.28-2.38] more days when they were limited by their physical or mental health. No statistically significant associations were noted among those who experienced one or two domains of racial discrimination compared with those who did not experience racial discrimination.

Compared with whites who had not experienced racial discrimination, the likelihood of poor physical health, poor mental health, or limitations from poor health were all higher among whites who experienced three or more domains of racial discrimination. For blacks, the RRs did not meet statistical significance for any of the four outcomes. There were associations among Latinos between racial discrimination and both poor mental health and days limited by poor health. Among Asian/PIs, a protective association [RR 0.29, 95\% CI 0.13-0.63] was noted between having experienced one or two domains of racial discrimination and physical health but this association was not observed for those who experienced three or more domains of racial discrimination.

Among the overall population, there was a significant interaction between exposure to racial discrimination and social relationships in the risk of experiencing poor mental health (Table 6). Among those exposed to racial discrimination, the likelihood for experiencing poor mental health was lower among those who had contact with family or friends outside their household at least once a week, compared with those who had less frequent social contact $(p=0.009)$. The association between exposure to racial discrimination and poor mental health is stratified by the social relationship variable in Table 7. A similar finding was noted for the outcome of days limited by mental or physical health. However, the interaction by social relationship status did not reach statistical significance $(p=0.08)$. There were no significant associations between the interaction of social contact and racial discrimination within any of the race/ethnicity groups analyzed separately.

\section{Discussion}

Overall, racial discrimination was associated with poor mental health among NYC's adult residential population. The strength of this association was lower among people who had frequent social contact. This study contributes to describing associations between exposure to racial discrimination and physical and mental health outcomes among a 


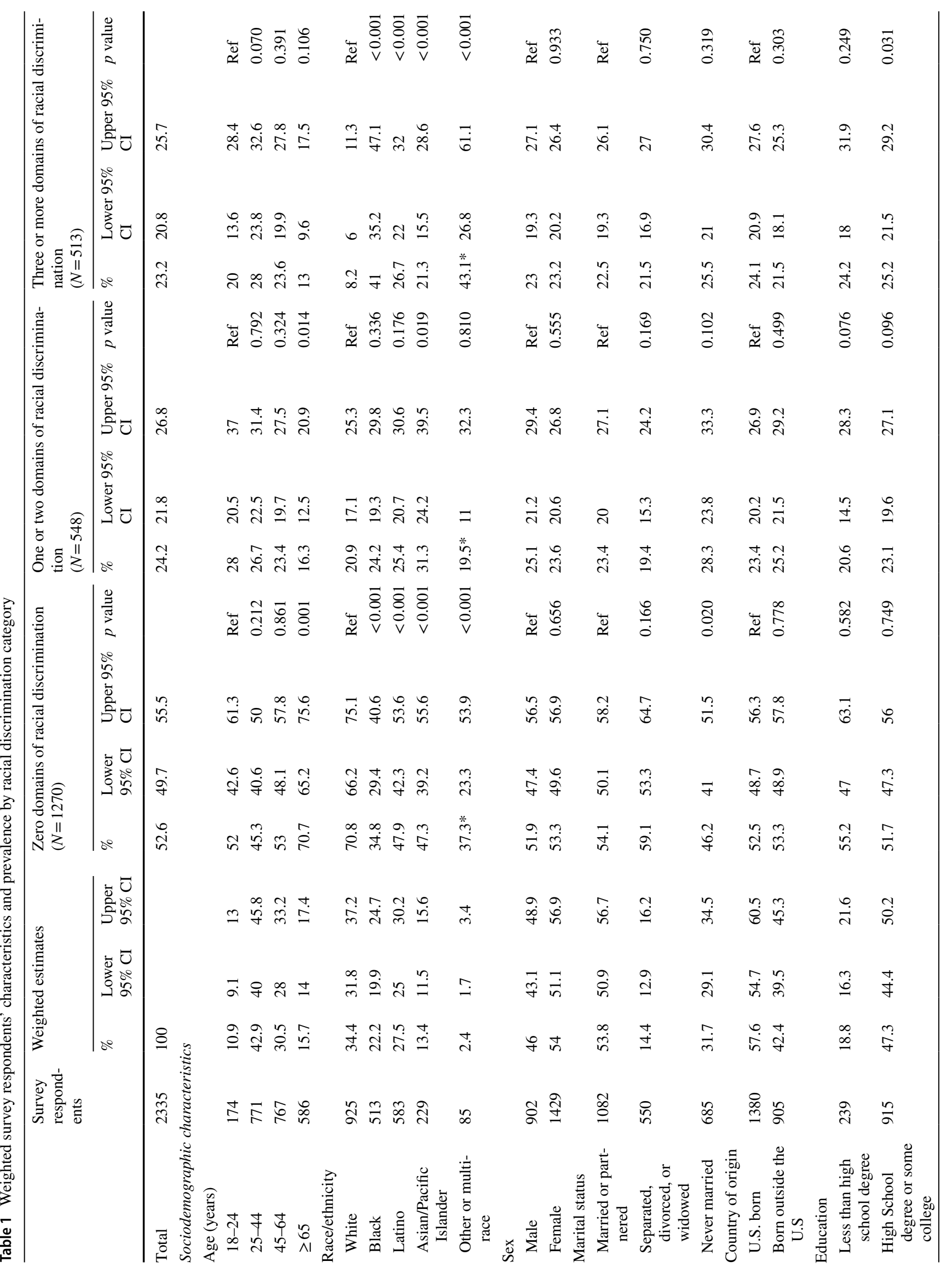




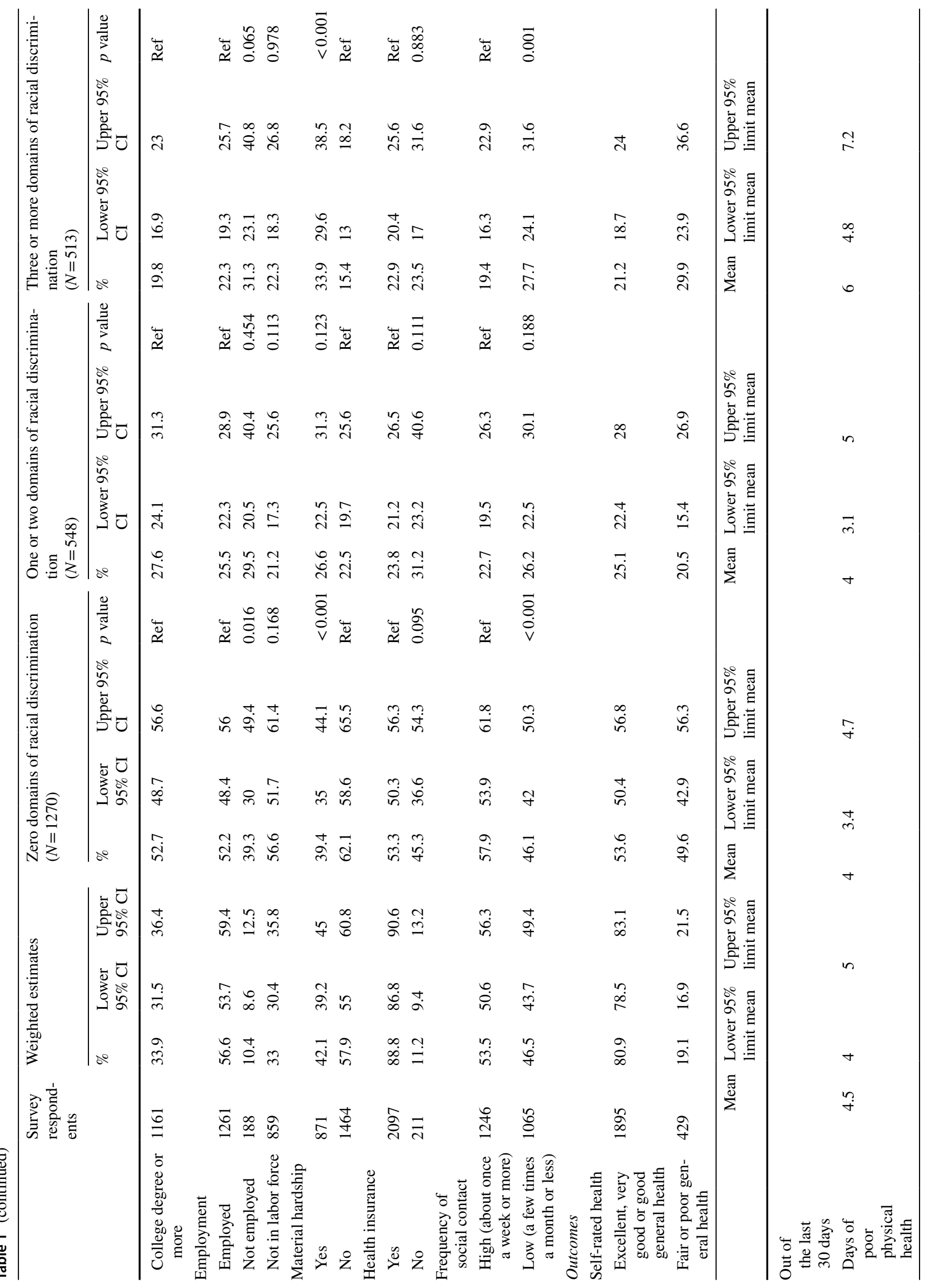




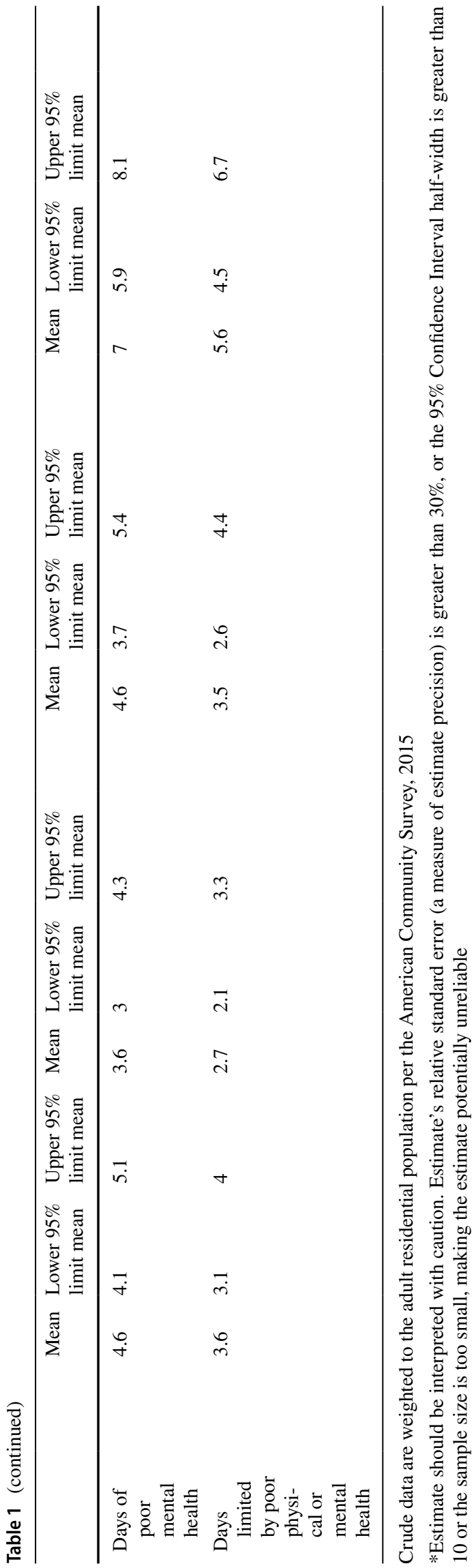

diverse urban population and provides evidence that social relationships could moderate that association. Exploring the moderating effect of social relationships on racial discrimination and health-related outcomes is of particular interest because it represents a modifiable behavior amenable to public health intervention.

The study findings generally echo the larger body of literature on racial discrimination and health. As previously documented, non-whites reported racial discrimination more frequently than whites [29, 30, 32]. Racial discrimination (including variations among race/ethnicity groups) was highly prevalent with approximately half $(47.4 \%$ [95\% CI $44.5,50.3]$ ) of respondents reporting experiencing at least one domain of racial discrimination in their lifetime. This figure is larger than in prior analyses of blacks and Latinos in four NYC neighborhoods in 2002 (17.3\% of blacks, $7.5 \%$ of Latinos) and in California in 2003-2005 (10.4\% of whites, $56.9 \%$ of blacks, $24-30.8 \%$ of Latinos) [32, 43]. However, it is lower than in a study in Chicago in 2003 (60-83\% of overall sample) [29], but generally similar to that observed in a unionized working adult sample in Boston in 2003-2004 ( $41.5 \%$ of whites, $66.6 \%$ of blacks, and $47.1 \%$ of Latinos) [33].

The literature generally reports a consistent association between exposure to racial discrimination and poor mental health and to a lesser degree with poor physical health $[7,24,44]$. Among a similar population to the one studied here, Stuber and colleagues reported that discrimination was associated with poor mental health, but not physical health among Latinos and blacks [43]. Similarly, Benjamins and colleagues reported in the Chicago study that racial discrimination was more consistently associated with mental than physical health outcomes [29]. We report again an association between racial discrimination and having more poor mental health days among residential New Yorkers, but also some significant associations for physical health outcomes.

We more frequently found significant associations between experiencing racial discrimination and poor health among those who had experienced three or more domains of racial discrimination than those who experienced one or two domains. This finding is consistent with prior literature indicating a dose-response association between exposure to racial discrimination and poor health [44].

Although blacks more frequently reported experiencing racial discrimination than whites, estimates for the association between exposure to racial discrimination and healthrelated quality of life outcomes did not reach statistical significance among blacks. In contrast, whites had significant associations between self-reported racial discrimination and poor health-related quality of life outcomes. People who identified as Middle Easterners and North Africans were categorized as white, but represented a relatively small percentage of the weighted population at $1.1 \%$ [95\% CI 0.6-2.0]. 
Table 2 Unadjusted and adjusted associations between exposure to racial discrimination and self-rated poor general health by race/ethnicity $(\mathrm{HrQOL} \# 1 *)$

\begin{tabular}{|c|c|c|c|c|c|c|c|c|}
\hline \multirow[t]{2}{*}{ Experiences of discrimination } & \multicolumn{4}{|l|}{ Unadjusted } & \multicolumn{4}{|l|}{ Adjusted } \\
\hline & Relative risk & Lower $95 \%$ CI & Upper $95 \%$ CI & $p$ value & Relative risk & Lower 95\% CI & Upper 95\% CI & $p$ value \\
\hline Overall population & \multicolumn{4}{|c|}{ Unadjusted $(N=2320)$} & \multicolumn{4}{|c|}{ Adjusted $(N=2241)$} \\
\hline 1 or 2 vs 0 & 0.90 & 0.65 & 1.26 & 0.5429 & 0.82 & 0.60 & 1.12 & 0.1957 \\
\hline$\geq 3$ vs 0 & 1.39 & 1.06 & 1.83 & 0.0209 & 1.15 & 0.87 & 1.52 & 0.3292 \\
\hline White & \multicolumn{4}{|c|}{ Unadjusted $(N=920)$} & \multicolumn{4}{|c|}{ Adjusted $(N=891)$} \\
\hline 1 or 2 vs 0 & 1.22 & 0.63 & 2.37 & 0.5599 & 0.83 & 0.44 & 1.57 & 0.5723 \\
\hline$\geq 3$ vs 0 & 2.96 & 1.64 & 5.36 & 0.0015 & 2.03 & 0.95 & 4.31 & 0.0833 \\
\hline Black & \multicolumn{4}{|c|}{ Unadjusted $(N=507)$} & \multicolumn{4}{|c|}{ Adjusted $(N=481)$} \\
\hline 1 or 2 vs 0 & 0.57 & 0.31 & 1.04 & 0.0615 & 0.57 & 0.31 & 1.05 & 0.0633 \\
\hline$\geq 3$ vs 0 & 0.85 & 0.52 & 1.38 & 0.5141 & 0.77 & 0.49 & 1.21 & 0.2505 \\
\hline Latino & \multicolumn{4}{|c|}{ Unadjusted $(N=580)$} & \multicolumn{4}{|c|}{ Adjusted $(N=567)$} \\
\hline 1 or 2 vs 0 & 1.00 & 0.60 & 1.66 & 0.9965 & 1.04 & 0.65 & 1.68 & 0.8601 \\
\hline$\geq 3$ vs 0 & 1.22 & 0.79 & 1.89 & 0.3800 & 1.25 & 0.84 & 1.86 & 0.2757 \\
\hline Asian/Pacific Islander & \multicolumn{4}{|c|}{ Unadjusted $(N=228)$} & \multicolumn{4}{|c|}{ Adjusted $(N=219)$} \\
\hline 1 or 2 vs 0 & 0.40 & 0.09 & 1.77 & 0.2120 & 0.42 & 0.11 & 1.69 & 0.1896 \\
\hline$\geq 3$ vs 0 & 0.66 & 0.25 & 1.72 & 0.3893 & 0.59 & 0.22 & 1.55 & 0.2831 \\
\hline
\end{tabular}

Data are weighted to the adult residential population per the American Community Survey, 2015

Adjusted relative risks are adjusted for age, sex, nativity, and material hardship

*Health-related Quality Of Life Question 1: "Would you say that in general your health is excellent, very good, good, fair or poor?"

Table 3 Unadjusted and adjusted associations between exposure to racial discrimination and days of poor physical health by race/ethnicity (HrQOL \#2*)

\begin{tabular}{|c|c|c|c|c|c|c|c|c|}
\hline \multirow[t]{2}{*}{ Experiences of discrimination } & \multicolumn{4}{|l|}{ Unadjusted } & \multicolumn{4}{|l|}{ Adjusted } \\
\hline & Relative risk & Lower $95 \%$ CI & Upper $95 \%$ CI & $p$ value & Relative risk & Lower $95 \%$ CI & Upper 95\% CI & $p$ value \\
\hline Overall population & \multicolumn{4}{|c|}{ Unadjusted $(N=2268)$} & \multicolumn{4}{|c|}{ Adjusted $(N=2194)$} \\
\hline 1 or 2 vs 0 & 1.00 & 0.76 & 1.32 & 0.9929 & 0.94 & 0.73 & 1.21 & 0.6497 \\
\hline$\geq 3$ vs 0 & 1.49 & 1.15 & 1.93 & 0.0025 & 1.40 & 1.08 & 1.80 & 0.0101 \\
\hline White & \multicolumn{4}{|c|}{ Unadjusted $(N=906)$} & \multicolumn{4}{|c|}{ Adjusted $(N=880)$} \\
\hline 1 or 2 vs 0 & 1.38 & 0.82 & 2.34 & 0.2294 & 1.01 & 0.64 & 1.58 & 0.9820 \\
\hline$\geq 3$ vs 0 & 2.31 & 1.46 & 3.66 & 0.0003 & 1.81 & 1.06 & 3.07 & 0.0283 \\
\hline Black & \multicolumn{4}{|c|}{ Unadjusted $(N=496)$} & \multicolumn{4}{|c|}{ Adjusted $(N=471)$} \\
\hline 1 or 2 vs 0 & 1.20 & 0.74 & 1.94 & 0.4615 & 1.20 & 0.73 & 1.95 & 0.4747 \\
\hline$\geq 3$ vs 0 & 1.49 & 0.85 & 2.60 & 0.1591 & 1.33 & 0.82 & 2.18 & 0.2508 \\
\hline Latino & \multicolumn{4}{|c|}{ Unadjusted $(N=560)$} & \multicolumn{4}{|c|}{ Adjusted $(N=548)$} \\
\hline 1 or 2 vs 0 & 0.94 & 0.59 & 1.50 & 0.8037 & 1.10 & 0.74 & 1.63 & 0.6409 \\
\hline$\geq 3$ vs 0 & 1.09 & 0.72 & 1.67 & 0.6791 & 1.35 & 0.92 & 1.97 & 0.1235 \\
\hline Asian/Pacific Islander & \multicolumn{4}{|c|}{ Unadjusted $(N=224)$} & \multicolumn{4}{|c|}{ Adjusted $(N=215)$} \\
\hline 1 or 2 vs 0 & 0.29 & 0.13 & 0.63 & 0.0019 & 0.29 & 0.14 & 0.59 & 0.0006 \\
\hline$\geq 3$ vs 0 & 1.17 & 0.53 & 2.58 & 0.6900 & 1.04 & 0.53 & 2.06 & 0.9075 \\
\hline
\end{tabular}

Data are weighted to the adult residential population per the American Community Survey, 2015

Adjusted relative risks are adjusted for age, sex, nativity, and material hardship

*Health-related Quality Of Life Question 2: "Now thinking about your physical health, which includes physical illness and injury, how many days during the past 30 days was your physical health not good?"

Similar findings have also been noted in other studies [29, 31]. The underlying reasons are unclear, but the Experiences of Discrimination measurement scale has previously been validated in racially diverse groups $[33,45]$. The impact of 
Table 4 Unadjusted and adjusted associations between exposure to racial discrimination and days of poor mental health by race/ethnicity (HrQOL \#3*)

\begin{tabular}{|c|c|c|c|c|c|c|c|c|}
\hline \multirow{2}{*}{$\begin{array}{l}\text { Experiences of } \\
\text { Discrimination }\end{array}$} & \multicolumn{4}{|l|}{ Unadjusted } & \multicolumn{4}{|l|}{ Adjusted } \\
\hline & Relative risk & $\begin{array}{l}\text { Lower } \\
95 \% \text { CI }\end{array}$ & $\begin{array}{l}\text { Upper } \\
95 \% \text { CI }\end{array}$ & $p$ value & Relative risk & $\begin{array}{l}\text { Lower } \\
95 \% \text { CI }\end{array}$ & $\begin{array}{l}\text { Upper } \\
95 \% \text { CI }\end{array}$ & $p$ value \\
\hline $\begin{array}{l}\text { Overall popula- } \\
\text { tion }\end{array}$ & Unadjusted $(N=2274)$ & & & & \multicolumn{4}{|c|}{ Adjusted $(N=2200)$} \\
\hline 1 or 2 vs 0 & 1.26 & 0.98 & 1.63 & 0.0768 & 1.16 & 0.88 & 1.53 & 0.3008 \\
\hline$\geq 3$ vs 0 & 1.93 & 1.52 & 2.46 & 0.0000 & 1.63 & 1.26 & 2.12 & 0.0002 \\
\hline White & Unadjusted $(N=905)$ & & & & \multicolumn{4}{|c|}{ Adjusted $(N=880)$} \\
\hline 1 or 2 vs 0 & 1.58 & 1.10 & 2.25 & 0.0124 & 1.40 & 0.94 & 2.10 & 0.1015 \\
\hline$\geq 3$ vs 0 & 2.50 & 1.63 & 3.85 & 0.0000 & 1.98 & 1.22 & 3.19 & 0.0053 \\
\hline Black & Unadjusted $(N=495)$ & & & & \multicolumn{4}{|c|}{ Adjusted $(N=469)$} \\
\hline 1 or 2 vs 0 & 1.64 & 0.81 & 3.33 & 0.1707 & 1.63 & 0.80 & 3.33 & 0.1827 \\
\hline$\geq 3$ vs 0 & 1.83 & 0.94 & 3.55 & 0.0758 & 1.57 & 0.77 & 3.22 & 0.2169 \\
\hline Latino & Unadjusted $(N=570)$ & & & & \multicolumn{4}{|c|}{ Adjusted $(N=558)$} \\
\hline 1 or 2 vs 0 & 0.85 & 0.50 & 1.45 & 0.5534 & 0.74 & 0.44 & 1.23 & 0.2379 \\
\hline$\geq 3$ vs 0 & 2.01 & 1.31 & 3.08 & 0.0013 & 1.66 & 1.09 & 2.53 & 0.0173 \\
\hline $\begin{array}{l}\text { Asian/Pacific } \\
\text { Islander }\end{array}$ & Unadjusted $(N=222)$ & & & & \multicolumn{4}{|c|}{ Adjusted $(N=213)$} \\
\hline 1 or 2 vs 0 & 1.21 & 0.51 & 2.86 & 0.6713 & 1.11 & 0.53 & 2.33 & 0.7893 \\
\hline$\geq 3$ vs 0 & 1.72 & 0.76 & 3.87 & 0.1931 & 1.43 & 0.63 & 3.27 & 0.3951 \\
\hline
\end{tabular}

Data are weighted to the adult residential population per the American Community Survey, 2015

Adjusted relative risks are adjusted for age, sex, nativity, and material hardship

*Health-related Quality Of Life Question 3: "Now thinking about your mental health, which includes stress, depression, and problems with emotions, how many days during the past 30 days was your mental health not good?"

Table 5 Unadjusted and adjusted associations between exposure to racial discrimination and days limited by poor physical or mental health by race/ethnicity (HrQOL \#4*)

\begin{tabular}{|c|c|c|c|c|c|c|c|c|}
\hline \multirow[t]{2}{*}{ Experiences of Discrimination } & \multicolumn{4}{|l|}{ Unadjusted } & \multicolumn{4}{|l|}{ Adjusted } \\
\hline & Relative risk & Lower 95\% CI & Upper $95 \% \mathrm{CI}$ & $p$ value & Relative risk & Lower 95\% CI & Upper $95 \%$ CI & $p$ value \\
\hline Overall population & \multicolumn{4}{|c|}{ Unadjusted $(N=2279)$} & \multicolumn{4}{|c|}{ Adjusted $(N=2206)$} \\
\hline 1 or 2 vs 0 & 1.32 & 0.95 & 1.85 & 0.1031 & 1.15 & 0.84 & 1.59 & 0.3798 \\
\hline$\geq 3$ vs 0 & 2.10 & 1.56 & 2.83 & 0.0000 & 1.74 & 1.28 & 2.38 & 0.0005 \\
\hline White & \multicolumn{4}{|c|}{ Unadjusted $(N=904)$} & \multicolumn{4}{|c|}{ Adjusted $(N=878)$} \\
\hline 1 or 2 vs 0 & 1.56 & 0.85 & 2.86 & 0.1540 & 1.10 & 0.67 & 1.82 & 0.7060 \\
\hline$\geq 3$ vs 0 & 3.80 & 2.52 & 5.71 & 0.0000 & 2.88 & 1.74 & 4.74 & 0.0000 \\
\hline Black & \multicolumn{4}{|c|}{ Unadjusted $(N=500)$} & \multicolumn{4}{|c|}{ Adjusted $(N=475)$} \\
\hline 1 or 2 vs 0 & 1.78 & 0.86 & 3.70 & 0.1232 & 1.75 & 0.81 & 3.81 & 0.1568 \\
\hline$\geq 3$ vs 0 & 2.14 & 1.10 & 4.19 & 0.0255 & 1.84 & 0.90 & 3.77 & 0.0967 \\
\hline Latino & \multicolumn{4}{|c|}{ Unadjusted $(N=568)$} & \multicolumn{4}{|c|}{ Adjusted $(N=557)$} \\
\hline 1 or 2 vs 0 & 1.16 & 0.67 & 2.03 & 0.5894 & 1.13 & 0.67 & 1.92 & 0.6387 \\
\hline$\geq 3$ vs 0 & 1.88 & 1.10 & 3.22 & 0.0212 & 1.80 & 1.11 & 2.91 & 0.0171 \\
\hline Asian/Pacific Islander & \multicolumn{4}{|c|}{ Unadjusted $(N=224)$} & \multicolumn{4}{|c|}{ Adjusted $(N=215)$} \\
\hline 1 or 2 vs 0 & 1.00 & 0.28 & 3.60 & 1.0000 & 0.92 & 0.34 & 2.51 & 0.8774 \\
\hline$\geq 3$ vs 0 & 1.81 & 0.49 & 6.70 & 0.3722 & 1.46 & 0.47 & 4.53 & 0.5082 \\
\hline
\end{tabular}

SDH data are weighted to the adult residential population per the American Community Survey, 2015

Adjusted relative risks are adjusted for age, sex, nativity, and material hardship

*Health-related Quality Of Life Question 4: "During the past 30 days, for about how many days did poor physical or mental health keep you from doing your usual activities, such as self-care, work, or recreation?" 


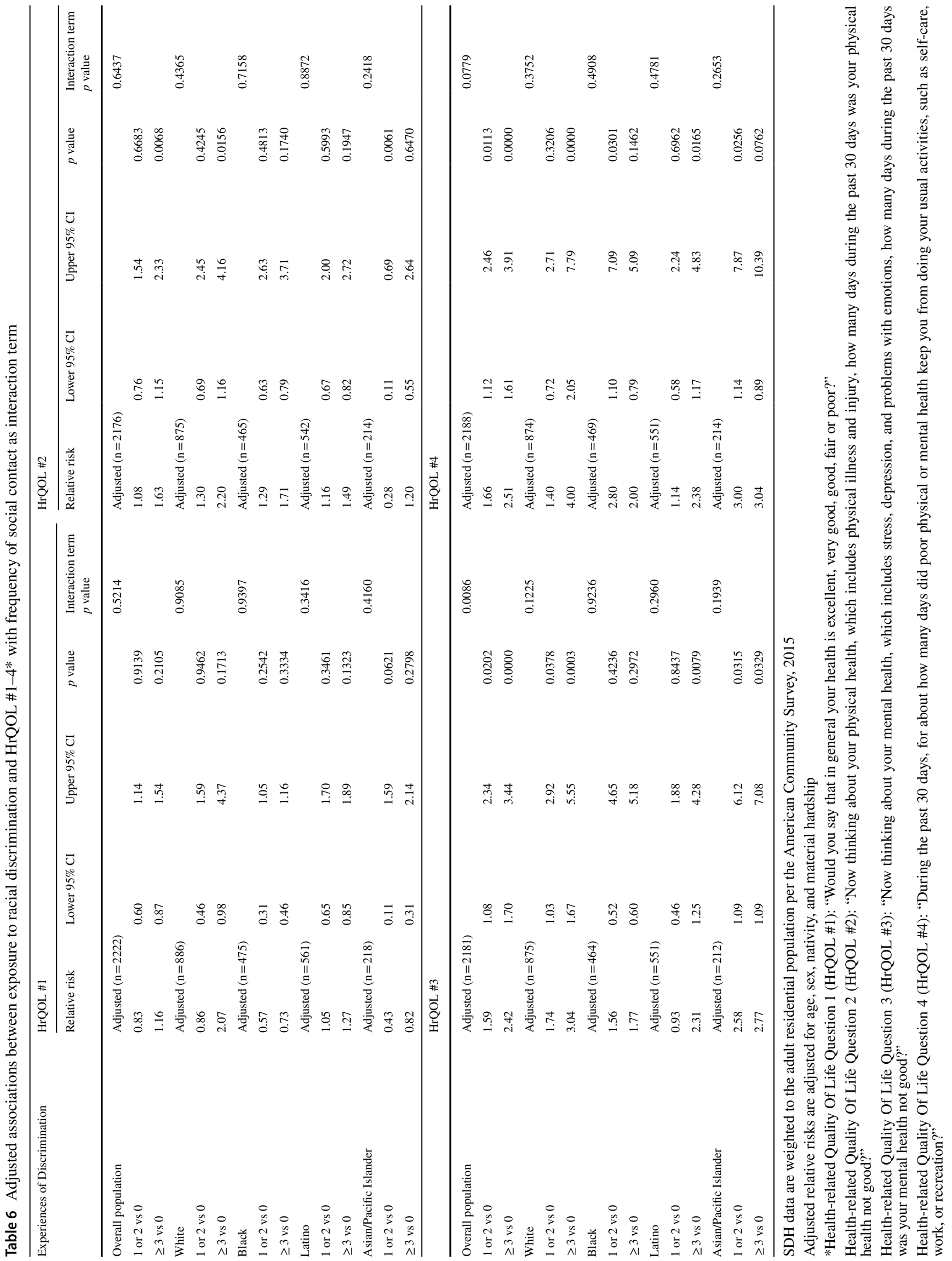


Table 7 Adjusted associations between exposure to racial discrimination and days of poor mental health (HrQOL \#3) in overall population stratified by frequency of social contact

\begin{tabular}{|c|c|c|c|c|c|c|c|c|}
\hline \multirow[t]{2}{*}{$\begin{array}{l}\text { Experiences of } \\
\text { discrimination }\end{array}$} & \multicolumn{4}{|c|}{$\begin{array}{l}\text { High-frequency social contact (about once a week or more) } \\
(N=997)\end{array}$} & \multicolumn{4}{|c|}{$\begin{array}{l}\text { Low-frequency social contact (a few times a month or less) } \\
(N=1184)\end{array}$} \\
\hline & Relative risk & Lower 95\% CI & Upper $95 \%$ CI & $p$ value & Relative risk & Lower 95\% CI & Upper $95 \%$ CI & $p$ value \\
\hline 1 or 2 vs 0 & 0.91 & 0.64 & 1.31 & 0.6135 & 1.54 & 1.02 & 2.31 & 0.0406 \\
\hline$\geq 3$ vs 0 & 1.24 & 0.90 & 1.72 & 0.1896 & 2.33 & 1.60 & 3.38 & 0.0000 \\
\hline
\end{tabular}

racial discrimination on health-related quality of life might differ by race/ethnicity.

We found a protective association between exposure to one or two domains of racial discrimination and physical health among Asian/PIs. This was an unexpected finding and inconsistent with previous literature [46]. This observation might be related to an unmeasured confounder that correlated with both reports of racial discrimination and health.

The findings should be interpreted in the context of its main limitations. First, a cross-sectional study design infers association, but not causation. Second, measuring racial discrimination is complex and there are ongoing controversies in the field of racial discrimination measurement [24]. Unmeasured personality traits and sociocultural factors influence how one experiences and frames prior experiences related to racial discrimination. Some respondents may minimize experiences of discrimination, whereas others may be hypervigilant. Different race/ethnicity groups might interpret questions related to health-related quality of life or racial discrimination differently. For instance, little is known about whether racial discrimination reported by whites can be compared with racial discrimination reported by non-majority groups. Additionally, older adults were more likely to have lived under institutionalized racial discriminatory practices. However, they reported experiencing fewer experiences of racial discrimination in their lifetime, compared with younger respondents. This result raises possible measurement validity questions regarding how older adults perceive and report events of racial discrimination in their lifetime. This finding might alternatively represent a survival bias where those who experienced more racial discrimination had poorer health and were not included in the survey possibly because of premature mortality or institutionalization. Third, we used broad categories for classifying race/ethnicity, but experiences can vary within groups. For instance, groups within Latinos might experience different levels of discrimination, but our sample size did not allow for further disaggregation [29]. Fourth, our study used a limited assessment of social relationships focused on the frequency of social contact, but did not ascertain quality or function of those relationships. Different domains of social relationships might have different influences on the association between discrimination and health. It is also important to recognize that strained social relationships can in fact undermine health [47]. Lastly, experiences of racial discrimination occur at the intersection of different axes of power and privilege (e.g., sex, economic opportunity, and intergenerational disparities) [48]. All those factors interact in complex ways in the social environment that are difficult to capture in a cross-sectional design.

The study findings underline opportunities for study and action. Further studies should qualitatively explore how people of different race/ethnicity, age, and economic standing experience, perceive, and report racial discrimination. Additionally, social relationship researchers have indicated that policies supporting social relationships could be construed as preventive medicine $[19,49]$. Such policies should also be considered in health equity and anti-racism policy agendas. Possible community interventions would be to create programs geared toward reducing social isolation such as safe spaces within communities where people can congregate and socialize $[50,51]$. Social relationships are a social determinant of health and public health departments should consider assessing them in more depth in community health surveys.

Disclaimer The findings and conclusions in this report are those of the authors and do not necessarily represent the official position of the Centers for Disease Control and Prevention.

\section{Compliance with ethical standards}

Conflict of interest The authors have no conflict of interest to declare.

\section{References}

1. Dolezsar, C. M., McGrath, J. J., Herzig, A. J. M., \& Miller, S. B. (2014). Perceived racial discrimination and hypertension: A comprehensive systematic review. Health Psychology, 33(1), 20.

2. Cozier, Y. C., Yu, J., Coogan, P. F., Bethea, T. N., Rosenberg, L., \& Palmer, J. R. (2014). Racism, segregation, and risk of obesity in the Black Women's Health Study. American Journal of Epidemiology, 179(7), 875-883.

3. Taylor, T. R., Williams, C. D., Makambi, K. H., Mouton, C., Harrell, J. P., Cozier, Y., et al. (2007). Racial discrimination and breast cancer incidence in US Black women: The Black Women's Health Study. American Journal of epidemIology, 166(1), 46-54.

4. Coogan, P. F., Yu, J., O'Connor, G. T., Brown, T. A., Cozier, Y. C., Palmer, J. R., et al. (2014). Experiences of racism and the 
incidence of adult-onset asthma in the Black Women's Health Study. Chest, 145(3), 480-485.

5. Brown, T. N., Williams, D. R., Jackson, J. S., Neighbors, H. W., Torres, M., Sellers, S. L., et al. (2000). "Being black and feeling blue": The mental health consequences of racial discrimination. Race and Society, 2(2), 117-131.

6. Barnes, L. L., Mendes De Leon, C. F., Lewis, T. T., Bienias, J. L., Wilson, R. S., \& Evans, D. A. (2008). Perceived discrimination and mortality in a population-based study of older adults. American Journal of Public Health, 98(7), 1241-1247.

7. Pascoe, E., \& Smart Richman, L. (2009). Perceived discrimination and health: A meta-analytic review. Psychological Bulletin, 135(4), 531-554.

8. Lewis, T. T., Aiello, A. E., Leurgans, S., Kelly, J., \& Barnes, L. L. (2010). Self-reported experiences of everyday discrimination are associated with elevated C-reactive protein levels in older AfricanAmerican adults. Brain, Behavior, and Immunity, 24(3), 438-443. https://doi.org/10.1016/j.bbi.2009.11.011.

9. Cunningham, T. J., Seeman, T. E., Kawachi, I., Gortmaker, S. L., Jacobs, D. R., Kiefe, C. I., et al. (2012). Racial/ethnic and gender differences in the association between self-reported experiences of racial/ethnic discrimination and inflammation in the CARDIA cohort of 4 US communities. Social Science and Medicine, 75(5), 922-931. https://doi.org/10.1016/j.socscimed.2012.04.027.

10. Fuller-Rowell, T. E., Doan, S. N., \& Eccles, J. S. (2012). Differential effects of perceived discrimination on the diurnal cortisol rhythm of African Americans and Whites. Psychoneuroendocrinology, 37(1), 107-118.

11. Brody, G. H., Lei, M. K., Chae, D. H., Yu, T., Kogan, S. M., \& Beach, S. R. (2014). Perceived discrimination among African American adolescents and allostatic load: A longitudinal analysis with buffering effects. Child Development, 85(3), 989-1002.

12. Geronimus, A. T., Hicken, M., Keene, D., \& Bound, J. (2006). "Weathering" and age patterns of allostatic load scores among blacks and whites in the United States. American Journal of Public Health, 96(5), 826-833.

13. Szanton, S. L., Rifkind, J. M., Mohanty, J. G., Miller, E. R., Thorpe, R. J., Nagababu, E., et al. (2012). Racial discrimination is associated with a measure of red blood cell oxidative stress: A potential pathway for racial health disparities. International Journal of Behavioral Medicine, 19(4), 489-495.

14. Chae, D. H., Nuru-Jeter, A. M., Adler, N. E., Brody, G. H., Lin, J., Blackburn, E. H., et al. (2014). Discrimination, racial bias, and telomere length in African-American men. American Journal of Preventive Medicine, 46(2), 103-111.

15. Beatty, D. L., Hall, M. H., Kamarck, T. A., Buysse, D. J., Owens, J. F., Reis, S. E., et al. (2011). Unfair treatment is associated with poor sleep in African American and Caucasian adults: Pittsburgh SleepSCORE project. Health Psychology, 30(3), 351-359.

16. Slopen, N., \& Williams, D. R. (2014). Discrimination, other psychosocial stressors, and self-reported sleep duration and difficulties. Sleep, 37(1), 147-156.

17. Borrell, L. N., Diez Roux, A. V., Jacobs, D. R., Jr., Shea, S., Jackson, S. A., Shrager, S., et al. (2010). Perceived racial/ethnic discrimination, smoking and alcohol consumption in the MultiEthnic Study of Atherosclerosis (MESA). Preventive Medicine, 51(3-4), 307-312. https://doi.org/10.1016/j.ypmed.2010.05.017.

18. Shariff-Marco, S., Klassen, A. C., \& Bowie, J. V. (2010). Racial/ ethnic differences in self-reported racism and its association with cancer-related health behaviors. American Journal of Public Health, 100(2), 364-374.

19. Holt-Lunstad, J., Smith, T. B., \& Layton, J. B. (2010). Social relationships and mortality risk: A meta-analytic review. PLoS Medicine, 7(7), e1000316.

20. Kawachi, I., \& Berkman, L. F. (2001). Social ties and mental health. Journal of Urban Health, 78(3), 458-467.
21. Valtorta, N. K., Kanaan, M., Gilbody, S., Ronzi, S., \& Hanratty, B. (2016). Loneliness and social isolation as risk factors for coronary heart disease and stroke: Systematic review and meta-analysis of longitudinal observational studies. Heart, 102(13), 1009-1016.

22. House, J. S., Landis, K. R., \& Umberson, D. (1988). Social relationships and health. Science, 241(4865), 540-545.

23. Cohen, S. (1988). Psychosocial models of the role of social support in the etiology of physical disease. Health Psychology, 7(3), 269.

24. Lewis, T. T., Cogburn, C. D., \& Williams, D. R. (2015). Selfreported experiences of discrimination and health: Scientific advances, ongoing controversies, and emerging issues. Annual Review of Clinical Psychology, 11(1), 407-440. https://doi. org/10.1146/annurev-clinpsy-032814-112728.

25. Brondolo, E., Brady, N., Pencille, M., Beatty, D., \& Contrada, R. J. (2009). Coping with racism: A selective review of the literature and a theoretical and methodological critique. Journal of Behavioral Medicine, 32(1), 64-88.

26. Valtorta, N. K., Kanaan, M., Gilbody, S., \& Hanratty, B. (2016). Loneliness, social isolation and social relationships: What are we measuring? A novel framework for classifying and comparing tools. British Medical Journal Open, 6(4), e010799.

27. Finch, B. K., \& Vega, W. A. (2003). Acculturation stress, social support, and self-rated health among Latinos in California. Journal of Immigrant Health, 5(3), 109-117.

28. American Association for Public Opinion Research (AAPOR) (2016). Standard definitions: Final dispositions of case codes and Outcome Rates for Surveys (9th ed.). Retrieved March 20, 2019, from https://www.aapor.org/.

29. Benjamins, M. R. (2013). Comparing measures of racial/ethnic discrimination, coping, and associations with health-related outcomes in a diverse sample. Journal of Urban Health, 90(5), 832-848. https://doi.org/10.1007/s11524-013-9787-x.

30. Benjamins, M. R. (2012). Race/ethnic discrimination and preventive service utilization in a sample of whites, blacks, Mexicans, and Puerto Ricans. Medical Care, 50(10), 870-876.

31. Ayalon, L., \& Gum, A. M. (2011). The relationships between major lifetime discrimination, everyday discrimination, and mental health in three racial and ethnic groups of older adults. Aging \& Mental Health, 15(5), 587-594.

32. Otiniano, A. D., \& Gee, G. C. (2012). Self-reported discrimination and health-related quality of life among Whites, Blacks, Mexicans and Central Americans. Journal of Immigrant and Minority Health, 14(2), 189-197.

33. Krieger, N., Smith, K., Naishadham, D., Hartman, C., \& Barbeau, E. M. (2005). Experiences of discrimination: Validity and reliability of a self-report measure for population health research on racism and health. Social Science and Medicine, 61(7), 1576-1596. https://doi.org/10.1016/j.socscimed.2005.03.006.

34. Centers for Disease Control and Prevention (2000). Measuring healthy days. Retrieved January 20, 2019, from https://www.cdc. gov/hrqol/pdfs/mhd.pdf.

35. Moriarty, D. G., Zack, M. M., \& Kobau, R. (2003). The centers for disease control and prevention's healthy days measures-population tracking of perceived physical and mental health over time. Health and Quality of Life Outcomes, 1(1), 37.

36. McCullagh, P. (1980). Regression models for ordinal data. Journal of the Royal Statistical Society: Series B (Methodological), 42(2), 109-127.

37. New York City Mayor's Office of Operations New York City Government Poverty Measure 2005-2015. Retrieved March 20, 2019, from https://nyc.gov/opportunity.

38. Coleman-Jensen, A., Rabbitt, M. P., Gregory, C. A., \& Singh, A. (2018). Household food security in the United States in 2017, ERR-256. US Department of Agriculture, Economic Research 
Service. Retrieved March 20, 2019, from https://www.ers.usda. gov.

39. United States Census Bureau (2016). Survey of income and program participation. Retrieved November 29, 2018, from https:// www.census.gov/programs-surveys/sipp/about.html.

40. Pearlin, L. I., Menaghan, E. G., Lieberman, M. A., \& Mullan, J. T. (1981). The stress process. Journal of Health and Social Behavior, 22(4), 337-356.

41. Williams, D. R., Yu, Y., Jackson, J. S., \& Anderson, N. B. (1997). Racial differences in physical and mental health: Socio-economic status, stress and discrimination. Journal of Health Psychology, 2(3), 335-351.

42. Maldonado, G., \& Greenland, S. (1993). Simulation study of confounder-selection strategies. American Journal of Epidemiology, 138(11), 923-936.

43. Stuber, J., Galea, S., Ahern, J., Blaney, S., \& Fuller, C. (2003). The association between multiple domains of discrimination and self-assessed health: A multilevel analysis of latinos and blacks in four low-income New York City neighborhoods. Health Services Research, 38(6, pt 2), 1735-1759. https://doi.org/10.111 1/j.1475-6773.2003.00200.x.

44. Paradies, Y. (2006). A systematic review of empirical research on self-reported racism and health. International Journal of Epidemiology, 35(4), 888-901.

45. Bastos, J. L., Celeste, R. K., Faerstein, E., \& Barros, A. J. (2010). Racial discrimination and health: A systematic review of scales with a focus on their psychometric properties. Social Science \& Medicine, 70(7), 1091-1099.

46. Gee, G. C., Ro, A., Shariff-Marco, S., \& Chae, D. (2009). Racial discrimination and health among Asian Americans: Evidence, assessment, and directions for future research. Epidemiologic Reviews, 31(1), 130-151.

47. Walen, H. R., \& Lachman, M. E. (2000). Social support and strain from partner, family, and friends: Costs and benefits for men and women in adulthood. Journal of Social and Personal Relationships, 17(1), 5-30.

48. Chetty, R., Hendren, N., Jones, M. R., \& Porter, S. (2018). Race and economic opportunity in the United States: An intergenerational perspective. Retrieved March 20, 2019, from https://www. equality-of-opportunity.org/assets/documents/race_slides.pdf.

49. Umberson, D., \& Karas Montez, J. K. (2010). Social relationships and health: A flashpoint for health policy. Journal of Health and Social Behavior, 51(Suppl), S54-S66.

50. Masi, C. M., Chen, H.-Y., Hawkley, L. C., \& Cacioppo, J. T. (2011). A meta-analysis of interventions to reduce loneliness. Personality and Social Psychology Review, 15(3), 219-266.

51. Dickens, A. P., Richards, S. H., Greaves, C. J., \& Campbell, J. L. (2011). Interventions targeting social isolation in older people: A systematic review. BMC Public Health, 11(1), 647.

Publisher's Note Springer Nature remains neutral with regard to jurisdictional claims in published maps and institutional affiliations. 